\title{
The Effect of Linear and Biweekly Non-Linear Periodized Resistance Training on Maximal Strength and Vertical Jump for Volleyball Players
}

\author{
Amr Hassan Tammam ${ }^{1}$ \\ Enas Mohamed Hashem ${ }^{2}$ \\ ${ }^{1}$ Professor of Sports Training at the Department of Training and Sports Movement Sciences, Faculty of Physical Education, \\ Tanta University, Egypt \\ ${ }^{2}$ Lecturer of Volleyball at the Department of Games and Racquet Sports, Faculty of Physical Education, Tanta University, \\ Egypt
}

\begin{abstract}
The purpose of this study was to verify the effect of a 12-weeks linear $(L P)$ and biweekly non-linear $(B W N L P)$ periodized resistance training program with equated volume and intensity on maximal strength and vertical jump (VJ) for volleyball players. Sixteen volleyball players of Tanta club with minimum 1-year strength training experience were divided into two groups: (LP, $n=8$ and BWNLP, $n=8$ ). They trained 4 days/ week in non-consecutive days using split routine. One repetition maximum (1RM) was measured during half squat, bench press, laying leg curl, seated arm curl, seated leg extension, and seated arm extension before training program (Pre), after 4 weeks of training (Mid-1), after 8 weeks of training (Mid-2), and after 12 weeks (Post). The results have shown statistically significant increase (p $\leq 0.05)$ in all exercises at MID-1, MID-2 and POST compared with PRE, for both LP and BWNLP models, statistically significant differences favor BWNLP $(p \leq 0.05)$ at POST for 4 exercises in comparison with the LP, and statistically significant differences favor BWNLP $(p \leq 0.05)$ at MID-2 for 2 exercises in comparison with the LP. No statistically significant differences ( $p>0.05$ ) between LP and BWNLP in maximal strength at MID-1. No statistically significant differences ( $p>0.05)$ between LP and BWNLP models in the MID-1, MID-2, and PRE evaluation for VJ test. In conclusion, the $B W N L P$ model outperformed the LP model making larger improvements in maximal strength, and a little higher percentage increase in VJ than LP model
\end{abstract}

Key words: Training, periodization, muscular strength

\section{Introduction}

$\mathrm{R}$ esistance training one of the methods is used to train lower body strength and power, to increase VJ (Newton, Rogers, Volek, Häkkinen, \& Kraemer, 2006). Presently, sport scientists are still trying to find the best system to develop athletes; however, they do not all agree on which strength training method is the most promising. Manipulating training variables in the most effective manner to increase strength can be a daunting task (Kraemer \& Ratamess, 2004).

Periodization is a training scheme where planned variations in training variables (e.g., number of sets and repetitions, exercise order, load, and rest) are manipulated in a manner that increases the ability of a person to achieve specific performance goals (e.g., strength) (Fleck, 1999; Rhea \& Alderman, 2004; Rhea, Ball, Phillips, \& Burkett, 2002). It is based on the overload principle and attempts to maximize the use of physical stress and recovery time by manipulating volume and intensity to facilitate important neuromuscular adaptations and to prevent the onset of overtraining syndrome (Prestes, De Lima, Frollini, Donatto, \& Conte, 2009).

There are two main models of periodization have been primary used by athletes and coaches, and examined in the literature. The first is the linear periodization (LP) is based on changing exercise volume and intensity across several mesocycles (3-4 months) to organize the training program (Brown \& Greenwood, 2005). Essentially, this type of exercise strategy starts with high volume and low intensity and then progresses to low-volume and high-intensity training, over a period of several months. The other main model is non-linear periodization (NLP) is based on the idea that volume and intensity are altered more frequently (daily, weekly or biweekly) by rotating different protocols to train various components of the neuromuscular system in order to give the neuromuscular system more frequent periods of recovery (Baker, Wilson, \& Carlyon, 1994). Daily non-linear periodization (DNLP), weekly non-linear periodization (WNLP), and biweekly non-linear periodization (BWNLP) were defined as a sub-type of NLP. The BWNLP is based on fluctuation of training 
variables occurs every two weeks (Baker et al., 1994; Rhea et al., 2002).

There are a multitude of studies, which have used DNLP (Monteiro et al., 2009; Peterson, Dodd, Alvar, Rhea, \& Favre, 2008; Rhea et al., 2003), and there are many studies that have been used WNLP (Baker et al., 1994; Buford, Rossi, Smith, \& Warren, 2007), the majority of studies have been conducted on untrained, or novices, or recreationally trained subjects, these studies did not yield any significant findings in favor of the DNLP or WNLP or BWNLP vs. LP. In the few studies examining competitive athletes, significant benefits of NLP training model compared with no periodization have been reported in football players (Hoffman et al., 2009; Kraemer, 1997), and significant benefits of WNLP training model compared with DNLP have been reported in volleyball players (Tammam, 2015).

Study of Baker et al. (Baker et al., 1994) is the only study which compared LP and BWNLP for strength gains, but in previously trained individuals, using mesocycle lasted 4 weeks in the LP program. The main differences between this study and the present study are that mesocycle in the present study for the LP program lasted 3 weeks, and participants were volleyball players. Thus, the study compared strength gains between BWNLP and LP programs with microcycles lasting 3 weeks in the LP program. Finally, to date there does not seem to be any research directly examining the effect of 12-weeks LP vs. BWNLP resistance training with equated volume and intensity on maximal strength and $\mathrm{VJ}$ for volleyball players. Knowledge gained through this study could help to make resistance training workouts more effective without having to alter volume or intensity. Thus, volleyball player could improve his workout without adding time or energy.

So the aim of current study was to verify the effect of a 12-weeks LP vs. BWNLP periodized resistance training program with equated volume and intensity on maximal strength and vertical jump (VJ) for volleyball players. Authors hypothesized that both LP and BWNLP will produce strength gains, and improve $\mathrm{VJ}$; furthermore the
BWNLP will produce greater strength gain and improve VJ than the LP.

\section{Material and Methods}

\section{Subjects}

Sixteen volleyball players from Tanta club minimum with minimum 1-year strength training experience volunteered to participate in current study. They were distributed in a balanced manner according to their Pre values of one repetition maximum (1RM) into equated two groups; (a) a group that performed 12 weeks of LP resistance training $(\mathrm{n}=8)$, and (b) a group that performed 12 weeks of BWNLP resistance training $(\mathrm{n}=8)$. The inclusion criteria for the participation were no use of any ergogenic supplements, not participate in any regular resistance training during the study, and If participants missed more than 2 training sessions, they were removed from the study. The 2 training groups showed no significant differences for pretraining characteristics ( $\mathrm{p}>0.05$ ) present in (Table 1). The study conformed to the Declaration of Helsinki guidelines for human use.

Table 1

Subjects' characteristics*

\begin{tabular}{|c|c|c|c|c|}
\hline \multirow{2}{*}{ Variables } & \multicolumn{2}{|c|}{ LP $(\mathrm{n}=8)$} & \multicolumn{2}{c|}{ BWNLP $(\mathrm{n}=8)$} \\
\cline { 2 - 5 } & Mean & \pm SD & Mean & \pm SD \\
\hline Age (y) & 24.75 & 2.49 & 25.13 & 3.27 \\
Weight $(\mathrm{kg})$ & 83.88 & 3.80 & 83.75 & 4.53 \\
Height (m) & 1.87 & 5.10 & 1.85 .5 & 4.99 \\
Body mass index (kg/ m2) & 24.10 & 1.16 & 24.33 & 0.60 \\
\hline
\end{tabular}

* $\mathrm{LP}=$ linear periodization; $\mathrm{BWNLP}=$ biweekly non-linear periodization.

\section{Experimental Procedures}

Timing of the study started 2 weeks after the end of the competitions, subjects were tested before training program (Pre), after 4 weeks of training (Mid-1), after 8 weeks of training (Mid-2), and after 12 weeks (Post) for VJ and $1 \mathrm{RM}$ of half squat, bench press, laying leg curl, seated arm curl, seated leg extension, and seated arm extension (Figure 1). 


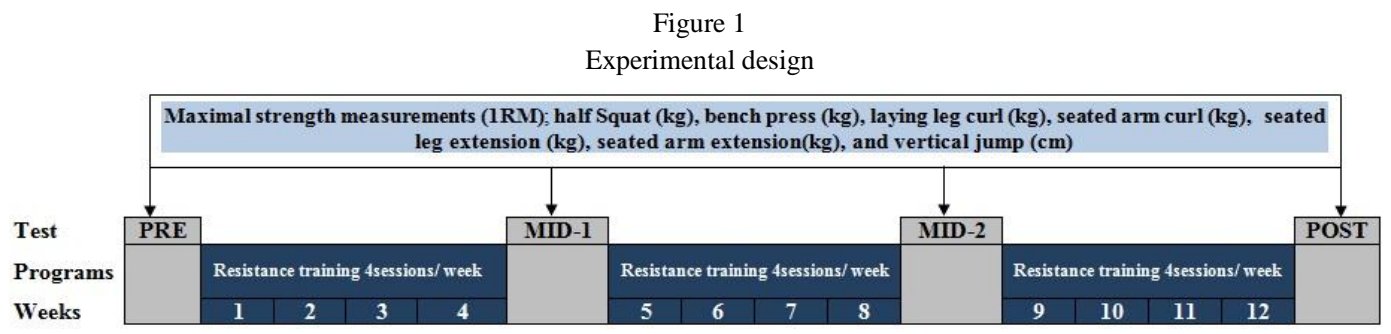

One week before the start of experiment, all subjects report to the Tanta club gym 4 times in non-consecutive days. On the 1st visit; all subjects were provided with an information sheet setting out details of the experiment, and written informed consent were completed and signed. They changed into sports clothes (running shoes, shorts and t-shirt), body weight and height were recorded using a balance weighing scales and portable stadiometer respectively. After warm up, subjects performed VJ test and completed a resistance training session with no or little resistance, this session familiarized the subjects with the equipments and proper exercise techniques. All testing and training sessions were supervised and conducted by the same authors, in addition to 2 certified fitness trainers.

\section{Maximal strength test}

$1 \mathrm{RM}$ was used as a measure of maximal strength. Two days after the 1st visit of familiarization session, all subjects performed 3 trails of 1RM on 3 separate days, with 48 hour between them for 6 exercises previously mentioned to determine the beginning loads $(\mathrm{kg}$ ) for subjects' resistance training programs, In order to facilitate the recovery and reduce the effect of fatigue, exercises were alternated between the upper and lower body. A high interclass correlation was found between the 2nd and 3rd $1 \mathrm{RM}$ trails $(\mathrm{R}=0.812)$. The greatest $1 \mathrm{RM}$ determined from the last two trails was used for PRE measurement. Before the test the subjects performed a warm-up which included 5 min of light treadmill running and 5 min stretching exercises. Thereafter, the subjects performed 10 repetitions at a relatively light load that served as a specific warm-up, followed by a gradual increase in load until 1RM was achieved (Baker et al., 1994). The rate of the gradual increase in load was dependent on the participant's self-perceived capacity, and it ranged from 1 to $10 \mathrm{~kg}$ for all exercises, with 3-5 min rest interval between attempts, and the 1RM was achieved within 3-5 attempts. The Authors provided encouragement to all subjects during testing, in an attempt to elicit a maximal effort. All testing sessions for 2 groups used the same test order, equipment, warm-up, and time of the tests (between 5:00 and 8:00 PM).

\section{Vertical jump test}

VJ height, defined as the difference between standing reach height and the maximal jump height was measured to the nearest $0.5 \mathrm{~cm}$ in all subjects using a countermovement jump with arm swing (Miller, Berry, Bullard, \& Gilders, 2002; Osternig, 1985). Briefly, the initial reach height of each subject was determined by having them stand, with feet flat, in a designated area adjacent to the wall with their dominant arm raised as high as possible. Each subject was then given an opportunity to perform 2-3 submaximal practice countermovement jumps. After a 2-3-min recovery, each subject performed three separate maximal VJ attempts with $30 \mathrm{sec}$ rest between attempts. The highest of the $3 \mathrm{VJ}$ attempts for each subject was utilized for data analysis. The interclass correlation coefficient for the VJ test trails were $(\mathrm{R}=$ 0.993).

\section{Resistance training program}

Resistance training programs were designed based on previous studies published in the literature in terms of periodization and duration (Apel, Lacey, \& Kell, 2011; Baker et al., 1994; Bradley-Popovich, 2001; Fleck \& Kraemer, 2014; Hartmann, Bob, Wirth, \& Schmidtbleicher, 2009; Kraemer et al., 2004; Kraemer et al., 1997; McNamara \& Stearne, 2010; Prestes, De Lima, et al., 2009). For 12 weeks of periodized resistance training (off-season period), subjects trained 4 sessions/ week in non-consecutive days, the program variables (e.g., intensity, total volume, rest intervals, repetition velocity, and exercise order) were the same for both the LP and BWNLP models. The difference between the two models was the sequence of training volume and intensity. In the LP model, training intensity was increased each mesocycle (3 weeks) and the volume was decreased, so the subjects trained with the same volumes and intensity for 3 weeks (Table2). In the BWNLP model, training intensity was modified every 2 weeks, so the subjects trained with the same volumes and intensity for 2 weeks. 
Table 2

Linear and biweekly non-linear periodized resistance training programs

\begin{tabular}{|c|c|c|c|c|c|c|c|}
\hline $\begin{array}{c}\text { LP: } \\
\text { Weeks } \\
4 \text { sessions / week }\end{array}$ & $\begin{array}{c}1-3 \\
3 \times 10 \mathrm{RM} \\
\end{array}$ & $\begin{array}{c}4-6 \\
3 \times 8 \mathrm{RM} \\
\end{array}$ & $\begin{array}{c}7-9 \\
3 \times 6 \mathrm{RM} \\
\end{array}$ & $\begin{array}{c}10-12 \\
3 \times 4 \mathrm{RM} \\
\end{array}$ & & & $\begin{array}{c}\text { Total Rep. } \\
1008\end{array}$ \\
\hline $\begin{array}{c}\text { BWNLP: } \\
\text { Weeks } \\
4 \text { sessions / week }\end{array}$ & $\begin{array}{c}1-2 \\
3 \times 10 R M\end{array}$ & $\begin{array}{c}3-4 \\
3 \times 8 R M\end{array}$ & $\begin{array}{c}5-6 \\
3 \times 4 R M\end{array}$ & $\begin{array}{c}7-8 \\
3 \times 10 R M\end{array}$ & $\begin{array}{c}9-10 \\
3 \times 6 R M\end{array}$ & $\begin{array}{c}11-12 \\
3 \times 4 R M\end{array}$ & $\begin{array}{c}\text { Total Rep. } \\
1008\end{array}$ \\
\hline
\end{tabular}

$\mathrm{LP}=$ linear periodization; $\mathrm{BWNLP}=$ biweekly non-linear periodization; $\mathrm{RM}=$ repetitions maximum; Total Rep. $=$ the total repetitions for Linear and biweekly non-linear periodization models over the study.

Split routine was used in the resistance training programs, training was divided into A (days 1 and 3) and B (days 2 and 4) in accordance with the recommendations of ACSM (Kraemer et al., 2002) for advanced athletes to train 4-6 days per week. The exercise order was strictly followed by both models, as presented in (Table 3 ). The repetition velocity of exercises and rest intervals between sets were according to training load and followed by both models, as presented in (Table 4).

Table 3

Exercises and training session sequences during LP and BWNLP programs*

\begin{tabular}{|c|c|}
\hline Training A (days 1 and 3) & Training B (days 2 and 4) \\
\hline Half squat & Bench press \\
Laying leg curl & Seated arm curl \\
Seated leg extension & Seated arm extension \\
\hline
\end{tabular}

* LP = linear periodization; BWNLP = biweekly non-linear periodization.

The training sessions consists of a warm-up period (10 $\mathrm{min}$ ), an exercise period (15-40 $\mathrm{min}$ ) according to session outcome, and a cool-down period (5 min). The warm-up and cool-down periods included stretching exercises and light treadmill running. Testing and Training sessions performed on 6 Panatta plate loaded machines, Italy. The MID-1 and MID-2 strength and VJ assessment were conducted after 48 hours from last session in 4th week and 8th week to establish a new load for the exercises in the next weeks, which enabled the subjects' bodies to be progressively overloaded throughout the 12 weeks. To calculate the weight $(\mathrm{Kg})$ of $(10,8,6$ and 4RM) according to 1RM load; Brzycki equation (Brzycki, 1995) was used $[1 \mathrm{RM}=\mathrm{W} \times 36 /(37-\mathrm{R})]$ where the $\mathrm{W}=$ weight $(\mathrm{Kg}) ; \mathrm{R}=$ number of repetitions ( 10 or 8 or 6 or 4 repetitions).
Table 4

Repetitions velocity and rest intervals according to training load

\begin{tabular}{|c|c|c|}
\hline $\begin{array}{c}\text { Training } \\
\text { load }\end{array}$ & Rep. Velocity & $\begin{array}{c}\text { Rest Intervals } \\
\text { (min) }\end{array}$ \\
\hline 10RM & $2: 1: 2$ & 3 \\
8RM & $1: 1: 2$ & 2 \\
$6 \mathrm{RM}$ & $1: 1: 1$ & 2 \\
4RM & $1: 1: 1$ & 1 \\
\hline
\end{tabular}

Rep. velocity = duration of concentric, pause, and eccentric phases of the movement; Rest intervals = rest between sets and exercises.

\section{Statistical analysis}

All data are presented as mean and standard deviations $( \pm$ SD). The statistical calculations were performed using IBM SPSS Statistics 22, (USA). All variables presented normal distribution and homoscedasticity. The repeatedmeasures analysis of variation (ANOVA) ( 2 models by 4 time points) was used to test for significant differences between groups, and when statistical significance $(\mathrm{P} \leq$ 0.05) was found, the Tukey HSD post hoc test for comparisons was applied to compare the strength gains between PRE, MID-1, MID-2, and POST time points. To test for significant differences between groups in pretraining variables, t-test was used. Test-retest reliability for $1 \mathrm{RM}$ and $\mathrm{VJ}$ were determined using an interclass correlation coefficient (ICC).

\section{Results}

There were no differences between the LP and BWNLP groups in the PRE test for all study variables ( $p>0.05)$. All subjects completed the 12 -week study with attendance rate more than $97.5 \%$.

Maximal Strength; there was a statistically significant increase $(\mathrm{p} \leq 0.05)$ in maximal strength for all exercises were noted at MID-1, MID-2 and POST compared with PRE evaluation, at MID-2 compared with MID-1 evaluation, and at POST compared with MID-2 evaluation for both LP and BWNLP models (Table 5). 
Table 5

Mean and \pm SD of Maximal strength variables and vertical jump at PRE, MID-1, MID-2 and POST for the LP and BWNLP models

\begin{tabular}{|c|c|c|c|c|}
\hline Exercise & PRE & MID-1 & MID-2 & POST \\
\hline \multicolumn{5}{|c|}{ Half squat $(\mathrm{kg})$} \\
\hline LP & $93.38(4.47)$ & $100.13(3.09) \dagger$ & $105.38(3.01) \dagger^{*}$ & $111.13(2.83) \dagger^{*} \$$ \\
\hline BWNLP & $93.88(2.64)$ & $103.50(2.33) \dagger$ & $110.38(3.42) \dagger * \#$ & $118(3.11) \dagger * \$ \#$ \\
\hline \multicolumn{5}{|c|}{ Bench press $(\mathrm{kg})$} \\
\hline LP & $57.38(3.11)$ & $61.75(2.19) \dagger$ & $64.38(2.13) \dagger^{*}$ & $67.75(2.49) \dagger * \$$ \\
\hline BWNLP & $58.88(3.91)$ & $64.38(3.16) \dagger$ & $69.88(3.09) \dagger * \#$ & $73(3.51) \dagger * \$ \#$ \\
\hline \multicolumn{5}{|c|}{ Laying leg curl $(\mathrm{kg})$} \\
\hline LP & $37.25(1.98)$ & $41.38(1.77) \dagger$ & $46.75(1.98) \dagger^{*}$ & $49.38(2.50) \dagger * \$$ \\
\hline BWNLP & $38(1.60)$ & $42.50(2.27) \dagger$ & $47.88(2.59) \dagger^{*} *$ & $51.25(2.60) \dagger * \$$ \\
\hline \multicolumn{5}{|c|}{ Seated arm curl $(\mathrm{kg})$} \\
\hline LP & $42.50(2.07)$ & $46.75(1.58) \dagger$ & $51.25(1.49) \dagger^{*}$ & $53.25(1.28) \dagger * \$$ \\
\hline BWNLP & $44.75(2.60)$ & $48.63(2.20) \dagger$ & $52.63(2.39) \dagger^{*}$ & $53.38(2.50) \dagger * \$$ \\
\hline \multicolumn{5}{|c|}{ Seated leg extension $(\mathrm{kg})$} \\
\hline LP & $41(2.07)$ & $46.25(2.25) \dagger$ & $49.13(2.95) \dagger^{*}$ & $52.13(2.95) \dagger * \$$ \\
\hline BWNLP & $42.38(1.77)$ & $47.13(2.17) \dagger$ & $51.88(1.89) \dagger^{*}$ & $56.88(1.81) \dagger * \$ \#$ \\
\hline \multicolumn{5}{|c|}{ Seated arm extension $(\mathrm{kg})$} \\
\hline LP & $40.25(1.28)$ & $44(1.77) \dagger$ & $47(2.14) \dagger^{*}$ & $50.50(1.60) \dagger * \$$ \\
\hline BWNLP & $42.13(1.96)$ & $46.13(2.30) \dagger$ & $49.38(1.13) \dagger^{*}$ & $54.13(2.90) \dagger * \$ \#$ \\
\hline \multicolumn{5}{|c|}{ Vertical jump $(\mathrm{cm})$} \\
\hline LP & $51(2.19)$ & $51.56(1.77)$ & $53.01(2.13)$ & $75(2.07) .53$ \\
\hline BWNLP & $51.13(2.55)$ & $51.87(1.98)$ & 53).53.22(2 & $54.36(2.74)$ \\
\hline
\end{tabular}

Values were expressed by mean \pm SD $(n=8$, each model), LP = Linear periodization; BWNLP = biweekly non-linear periodization; $\mathrm{PRE}=$ baseline evaluation; $\mathrm{MID}-1=$ evaluation in the end of 4 th week of training; MID-2 = evaluation in the end of 8th week of training; POST = evaluation after 12 weeks of training, †statistically significant difference in comparison with PRE, *statistically significant difference in comparison with MID-1, \$statistically significant difference in comparison with MID-2, \#statistically significant difference in comparison with LP $(\mathrm{p} \leq 0.05)$.

The study has shown statistically significant differences favor BWLNP model $(p \leq 0.05)$ at the POST evaluation in half squat strength of $25.70 \%$, bench press strength of $23.99 \%$, in seated leg extension strength of $34.22 \%$, and in arm extension strength of $28.49 \%$ in comparison with the LP model (19.01\%, 18.08\%, 27.13\% and $25.47 \%$; respectively), whereas there is no statistically significant differences ( $\mathrm{p}>0.05$ ) were found between LP and BWNLP in laying leg curl and seated arm curl at POST evaluation. Results also showed statistically significant differences favor BWLNP model $(\mathrm{p} \leq 0.05)$ at the MID-2 evaluation in half squat strength of $17.58 \%$, and bench press strength of $18.69 \%$, in comparison with the LP model $(12.85 \%$ and $12.20 \%$; respectively), whereas there is no statistically significant differences $(p>0.05)$ were found between LP and BWLNP in the rest of exercise at MID-2 evaluation. Also the study has shown no statistically significant differences $(p>0.05)$ between LP and BWNLP in all exercises at MID-1 evaluation, although no statistically significant differences $(p>0.05)$ were found between LP and BWNLP in maximal strength at MID-1, MID-2 for 4 exercises, and POST for 2 exercises, however the BWNLP model shown a higher percentage increase in maximal strength than LP model for those exercises (Table 5) (Fig. 2). 
Figure 2

Rate of increase in maximal strength for LP and BWNLP models

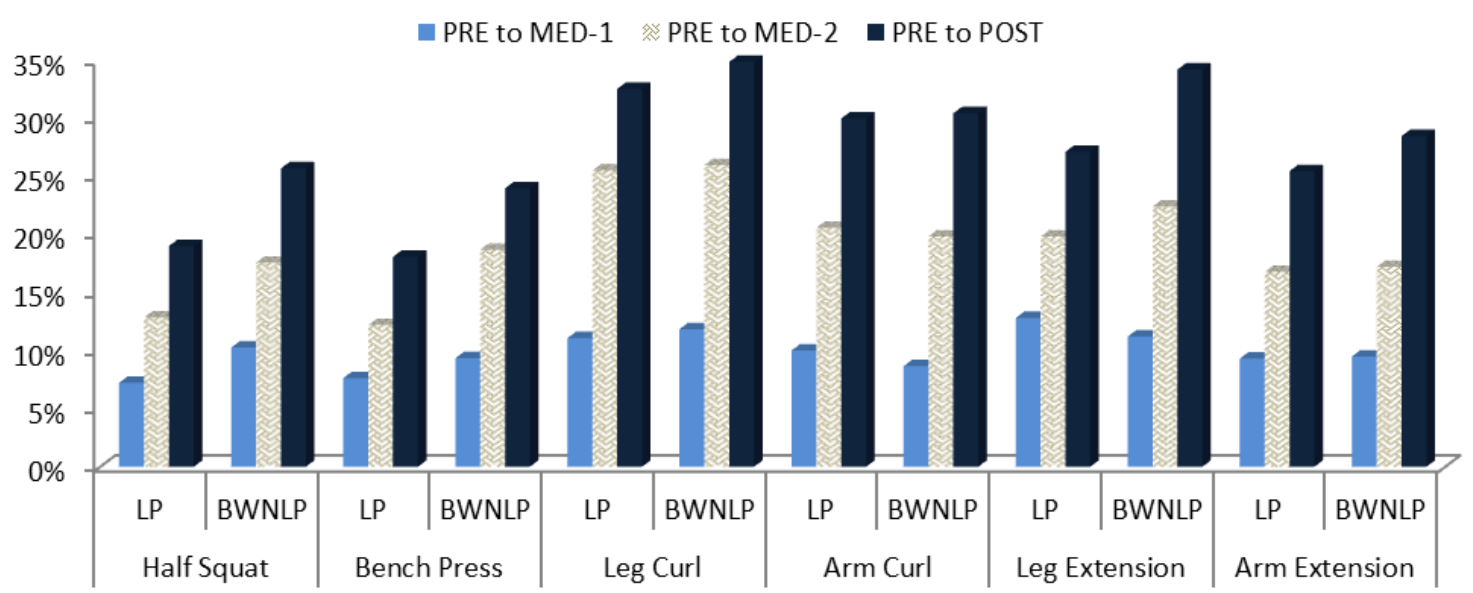

In LP model, rates of increase in maximal strength ranged between $18.08 \%$ in bench press and $32.55 \%$ in seated leg curl at the end of the training program, while in BWNLP model rates of increase in maximal strength ranged between $23.99 \%$ in bench press and $34.87 \%$ in seated leg curl at the end of the training program (Fig. 2)
Vertical Jump; the study has shown no statistically significant differences $(\mathrm{p}>0.05)$ between LP and BWNLP models in the MID-1, MID-2, and PRE evaluation for VJ test, however the BWNLP model shown a higher percentage increase in $\mathrm{VJ}$ than LP model ( $\mathrm{LP}=5.39 \%$; BWNLP $=6.32 \%)($ Table 5) (Fig. 3).

Figure 3

Rate of increase in vertical jump for LP and BWNLP models

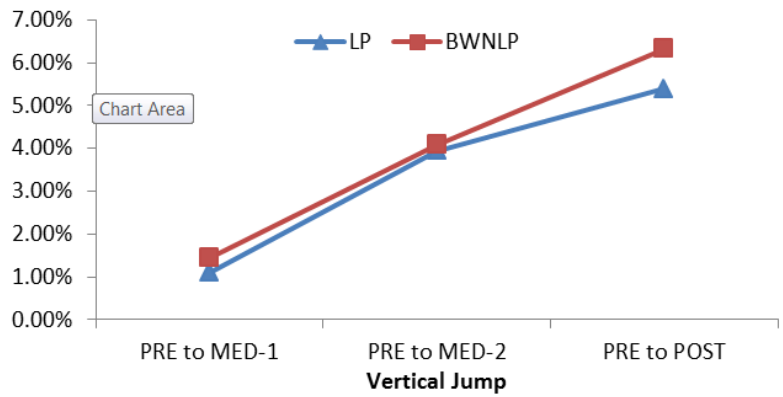

\section{Discussion}

\section{Maximal Strength}

Results have shown that both the LP and BWNLP programs caused significant increases in muscular strength of all exercises at PRE, MID-1, MID-2, and POST evaluation in volleyball players. Another interesting aspect was the LP and BWNLP programs caused a higher muscular strength gains at MID-1, compared with muscular strength gains at MID-2, and compared with muscular strength gains at POST evaluation in all exercises. These results indicate that LP and BWNLP models may increase maximal strength to a greater extent during the initial training period and result in more consistent strength gains as training progresses. These observations are supported by the results of previous studies (Apel et al., 2011; Baker et al., 1994; Kraemer et al., 2004; Kraemer \& Ratamess, 2005; Prestes, Frollini, et al., 2009; Rhea et al., 2003). The initial strength gains (14 weeks) due to resistance training are primarily neural adaptations such as: 1) Increased motor unit recruitment, 2) Increased firing frequency, 3) Increased synchronicity of firing and/or 4) decreased co-contraction of the antagonist muscle (Baechle \& Earle, 2000; Behm, 1995; Häkkinen, Pakarinen, \& Kallinen, 1992; Kraemer \& Häkkinen, 2008), after this period strength gains are also influenced by increases in muscle mass (Brandenburg \& Docherty, 2002; Deschenes \& Kraemer, 2002; Gearhart JR et al., 2001). The greater increases in muscular strength with BWNLP compared to LP during the first weeks of training indicate that BWNLP may induce quicker neural adaptations than LP model. Thus we partially support our 
first hypothesis that both LP and BWNLP periodized training models will produce strength gains. One of the most important finding of this study was that there were no significant $(p>0.05)$ differences in strength between the LP and BWNLP models in MID-1 evaluation, but at the end of training programs, the BWNLP model was significantly $(\mathrm{p} \leq 0.05)$ stronger than the LP model in 4of 6 exercises (i.e., half squat, bench press, seated leg extension, and seated arm extension), while no significant between LP and BWNLP models in the other 2 exercises. The suggests that to elicit strength increases, the choice of training model may become important at some point after week 4 , and it may be related to the biweekly order of intensity. The LP model increased strength by an average between $18.08 \%$ and $32.55 \%$ at POST, whereas the BWNLP model increased strength by an average between $23.99 \%$ and $34.87 \%$ at POST. Thus, BWNLP strength training program was more successful at increasing muscular strength by week 12 than was LP strength training program. That results have not confirmed with study of Baker et al. (Baker et al., 1994) when compared LP strength training with BWNLP strength training and found no significant $(p>0.05)$ differences between the 2 periodization models in the development of strength. Thus we partially support our second hypothesis that the BWNLP will produce greater strength gain than the LP.

Comparing the current study with other periodized strength training studies is not straightforward for many aspects, the most important aspect, some of studies (Baker et al., 1994; Buford et al., 2007), and the present study all equated the training volume between the groups. However, another studies (Hoffman et al., 2009; Rhea et al., 2002) did not equate training volume between the linear and non-linear programs. Another important difference among these studies pertains to the NLP program. The studies of Buford et al. (Buford et al., 2007), Hoffman et al. (Hoffman et al., 2009), and Rhea et al. (Rhea et al., 2002) used NLP, with a 3 sessions/ week schedule, the current study used a 4 sessions/ week schedule, and the intensity changed dramatically every 2 weeks and not in a progressively increasing manner. Another important difference among these studies pertains to subjects, the studies of Buford et al. (Buford et al., 2007), Hoffman et al. (Hoffman et al., 2009), and Rhea et al. (Rhea et al., 2002) have been conducted on untrained, or recreationally trained subjects, these studies did not yield any significant findings in favor of the NLP vs. LP, the current study was conducted on volleyball players have experience in strength training. Another interesting comparison is the magnitude of strength gains produced by using whole body routines and split routines. Our training protocol used split routines, whereas, Buford et al. (Buford et al., 2007), Hoffman et al. (Hoffman et al.,
2009), and Rhea et al. (Rhea et al., 2002) used whole body routines. Split routines would facilitate recovery due to the alternation in the muscle group trained. Thus, the variation in results among these studies is likely related to some combination of total training volume (e.g., days per week), weight training experience or LP vs. NLP models, or whole body vs. split routines, or all.

\section{Vertical Jump}

Results have shown that both the LP and BWNLP programs caused non-significant increases in $\mathrm{VJ}$ of all time evaluations, however the BWNLP model shown a little higher percentage increase in VJ than LP model, and comparable in magnitude to that reported by other researchers using strength training (Adams, O'Shea, O'Shea, \& Climstein, 1992; Baker et al., 1994). This suggests that improvements in maximal strength do not necessarily equate to improvements in power activities, such as jumping. The reasons for this are not clear and definitely warrant further investigations.

\section{Conclusion}

In conclusion, the present study's findings indicate that both LP and BWNLP periodized training programs made strength gains in the end of program; however BWNLP resistance training results more gains in maximal strength, when training volume and relative intensity are equated, the BWNLP has a little higher percentage increase in VJ than LP model.

\section{References}

1. Adams, K., O'Shea, J. P., O'Shea, K. L., \& Climstein, M. (1992). The effect of six weeks of squat, plyometric and squat-plyometric training on power production. The Journal of Strength \& Conditioning Research, 6(1), 36-41.

2. Apel, J. M., Lacey, R. M., \& Kell, R. T. (2011). A comparison of traditional and weekly undulating periodized strength training programs with total volume and intensity equated. The Journal of Strength \& Conditioning Research, 25(3), 694-703.

3. Baechle, T. R., \& Earle, R. W. (2000). Essentials of strength training and conditioning: National strength and conditioning association. Champaign, IL: Human Kinetics.

4. Baker, D., Wilson, G., \& Carlyon, R. (1994). Periodization: The Effect on Strength of Manipulating Volume and Intensity. The Journal of Strength \& Conditioning Research, 8(4), 235-242.

5. Behm, D. G. (1995). Neuromuscular implications and applications of resistance training. The Journal of Strength \& Conditioning Research, 9(4), 264-274. 
6. Bradley-Popovich, G. E. (2001). Nonlinear versus linear periodization models. Strength \& Conditioning Journal, 23(1), 42.

7. Brandenburg, J. E., \& Docherty, D. (2002). The effects of accentuated eccentric loading on strength, muscle hypertrophy, and neural adaptations in trained individuals. The Journal of Strength \& Conditioning Research, 16(1), 25-32.

8. Brown, L. E., \& Greenwood, M. (2005). Periodization essentials and innovations in resistance training protocols. Strength \& Conditioning Journal, 27(4), 80-85.

9. Brzycki, M. (1995). A practical approach to strength training: Masters Press Indianapolis, IN.

10. Buford, T. W., Rossi, S. J., Smith, D. B., \& Warren, A. J. (2007). A comparison of periodization models during nine weeks with equated volume and intensity for strength. The Journal of Strength \& Conditioning Research, 21(4), 1245-1250.

11. Deschenes, M. R., \& Kraemer, W. J. (2002). Performance and physiologic adaptations to resistance training. American Journal of Physical Medicine \& Rehabilitation, 81(11), S3-S16.

12. Fleck, S. J. (1999). Periodized strength training: a critical review. The Journal of Strength \& Conditioning Research, 13(1), 82-89.

13. Fleck, S. J., \& Kraemer, W. (2014). Designing Resistance Training Programs, 4E: Human Kinetics.

14. Gearhart JR, R. F., Goss, F. L., LAGALLY, K. M., JAKICIC, J. M., GALLAGHER, J., \& ROBERTSON, R. J. (2001). Standardized scaling procedures for rating perceived exertion during resistance exercise. The Journal of Strength \& Conditioning Research, 15(3), 320-325.

15. Häkkinen, K., Pakarinen, A., \& Kallinen, M. (1992). Neuromuscular adaptations and serum hormones in women during short-term intensive strength training. European journal of applied physiology and occupational physiology, 64(2), 106-111.

16. Hartmann, H., Bob, A., Wirth, K., \& Schmidtbleicher, D. (2009). Effects of different periodization models on rate of force development and power ability of the upper extremity. The Journal of Strength \& Conditioning Research, 23(7), 1921-1932.

17. Hoffman, J. R., Ratamess, N. A., Klatt, M., Faigenbaum, A. D., Ross, R. E., Tranchina, N. M., . . . Kraemer, W. J. (2009). Comparison between different offseason resistance training programs in Division III American college football players. The Journal of Strength \& Conditioning Research, 23(1), 11-19.

18. Kraemer, W. J. (1997). A Series of Studies-The Physiological Basis for Strength Training in American
Football: Fact Over Philosophy. The Journal of Strength \& Conditioning Research, 11(3), 131-142.

19. Kraemer, W. J., Adams, K., Cafarelli, E., Dudley, G. A., Dooly, C., Feigenbaum, M. S., . . . Hoffman, J. R. (2002). American College of Sports Medicine position stand. Progression models in resistance training for healthy adults. Medicine and science in sports and exercise, 34(2), 364-380.

20. Kraemer, W. J., \& Häkkinen, K. (2008). Handbook of Sports Medicine and Science, Strength Training for Sport: John Wiley \& Sons.

21. Kraemer, W. J., Nindl, B. C., Ratamess, N. A., Gotshalk, L. A., Volek, J. S., Fleck, S. J., . . Hakkinen, K. (2004). Changes in muscle hypertrophy in women with periodized resistance training. Medicine and science in sports and exercise, 36(4), 697-708.

22. Kraemer, W. J., \& Ratamess, N. A. (2004). Fundamentals of resistance training: progression and exercise prescription. Medicine and science in sports and exercise, 36(4), 674-688.

23. Kraemer, W. J., \& Ratamess, N. A. (2005). Hormonal responses and adaptations to resistance exercise and training. Sports Medicine, 35(4), 339-361.

24. Kraemer, W. J., Volek, J. S., Clark, K. L., Gordon, S. E., Incledon, T., Puhl, S. M., . . . Sebastianelli, W. J. (1997). Physiological adaptations to a weight-loss dietary regimen and exercise programs in women. Journal of applied physiology, 83(1), 270-279.

25. McNamara, J. M., \& Stearne, D. J. (2010). Flexible nonlinear periodization in a beginner college weight training class. The Journal of Strength \& Conditioning Research, 24(8), 2012-2017.

26. Miller, M. G., Berry, D. C., Bullard, S., \& Gilders, R. (2002). Comparisons of land-based and aquatic-based plyometric programs during an 8-week training period. Journal of Sport Rehabilitation, 11(4), 268-283.

27. Monteiro, A. G., Aoki, M. S., Evangelista, A. L., Alveno, D. A., Monteiro, G. A., da Cruz Piçarro, I., \& Ugrinowitsch, C. (2009). Nonlinear periodization maximizes strength gains in split resistance training routines. The Journal of Strength \& Conditioning Research, 23(4), 1321-1326.

28. Newton, R. U., Rogers, R. A., Volek, J. S., Häkkinen, K., \& Kraemer, W. J. (2006). Four weeks of optimal load ballistic resistance training at the end of season attenuates declining jump performance of women volleyball players. The Journal of Strength \& Conditioning Research, 20(4), 955-961. 
29. Osternig, L. R. (1985). Isokinetic dynamometry: implications for muscle testing and rehabilitation. Exercise and sport sciences reviews, 14, 45-80.

30. Peterson, M. D., Dodd, D. J., Alvar, B. A., Rhea, M. R., \& Favre, M. (2008). Undulation training for development of hierarchical fitness and improved firefighter job performance. The Journal of Strength \& Conditioning Research, 22(5), 1683-1695.

31. Prestes, J., De Lima, C., Frollini, A. B., Donatto, F. F., \& Conte, M. (2009). Comparison of linear and reverse linear periodization effects on maximal strength and body composition. The Journal of Strength \& Conditioning Research, 23(1), 266-274.

32. Prestes, J., Frollini, A. B., de Lima, C., Donatto, F. F., Foschini, D., de Cássia Marqueti, R., . . . Fleck, S. J. (2009). Comparison between linear and daily undulating periodized resistance training to increase strength. The Journal of Strength \& Conditioning Research, 23(9), 2437-2442.
33. Rhea, M. R., \& Alderman, B. L. (2004). A metaanalysis of periodized versus nonperiodized strength and power training programs. Research quarterly for exercise and sport, 75(4), 413-422.

34. Rhea, M. R., Ball, S. D., Phillips, W. T., \& Burkett, L. N. (2002). A comparison of linear and daily undulating periodized programs with equated volume and intensity for strength. The Journal of Strength \& Conditioning Research, 16(2), 250-255.

35. Rhea, M. R., Phillips, W. T., BURKETT, L. N., STONE, W. J., BALL, S. D., ALVAR, B. A., \& THOMAS, A. B. (2003). A comparison of linear and daily undulating periodized programs with equated volume and intensity for local muscular endurance. The Journal of Strength \& Conditioning Research, 17(1), 82-87.

36. Tammam, A. H., Hashem, Enas M. . (2015). Comparison between Daily and Weekly Undulating Periodized Resistance Training to Increase Muscular Strength for Volleyball Players. Journal of Applied Sports Science, 5(3), 725-744. 